\title{
Cold Feedback in Cooling-Flow Galaxy Clusters
}

\author{
Fabio Pizzolato \\ Department of Physics \\ Technion-Israel Institute of Technology \\ Haifa 32000 \\ fabio@physics.technion.ac.il
}

Summary. We put forward an alternative view to the Bondi-driven feedback between heating and cooling of the intra-cluster medium (ICM) in cooling flow (CF) galaxies and clusters.

We adopt the popular view that the heating is due to an active galactic nucleus (AGN), i.e. a central black hole accreting mass and launching jets and/or winds. We propose that the feedback occurs with the entire cool inner region $(r \lesssim 5-30 \mathrm{kpc})$. A moderate cooling flow does exist here, and non-linear over-dense blobs of gas cool fast and are removed from the ICM before experiencing the next major AGN heating event. Some of these blobs may not accrete on the central black hole, but may form stars and cold molecular clouds. We discuss the conditions under which the dense blobs may cool to low temperatures and feed the black hole.

\section{Introduction}

The observations of galaxy clusters with the last generation X-ray satellites Chandra and XMM-Newton failed to detect the large amounts of cool gas predicted by the old version (Fabian, 1994) of the cooling flow (CF) model (see e.g. Peterson \& Fabian, 2006 for a recent review). The most straightforward explanation is that the intra-cluster medium (ICM) in CF clusters is heated by some mechanism, the currently most popular candidate being the active galactic nucleus (AGN) residing at the core of the cluster dominant galaxy.

Most models of AGN heating agree in that there is some feedback between the heating and the radiative cooling, possibly resulting in intermittent AGN activity. There are two approaches to the AGN/ICM feedback. In the hot feedback scenario (see e.g. Nulsen, 2004) the ICM never cools below X-ray emitting temperatures; the AGN accretion pattern is Bondi-like, and is determined by the ICM properties at the Bondi radius, which is typically few tens pc.

In the second approach, dubbed cold feedback, (see Pizzolato \& Soker, 2005 and Soker, 2006 for more details), the black hole accretes cold gas, although the amount of cooling mass is much below that predicted by the old cooling flow model. In this case the feedback takes place within a region extending to a distance of $\approx 5-30 \mathrm{kpc}$ from the cluster centre. 


\section{The Cold Feedback Scenario}

The cold feedback scenario entails a cycle in the cooling/accretion activity.

We suggest that this cycle starts with a major AGN outburst, which injects a huge amount of energy into the ICM. The AGN outburst interacts in a very complicated fashion with the ICM (Begelman, 2004), e.g. it heats and inflates radio bubbles, and may also stir some turbulence. The ICM itself is displaced and thickened by the rising bubbles, as shown by their rims' enhanced X-ray brightness (Blanton et al., 2001). A non-homogeneous thickening may result in the formation of a multi-phase gas, consisting e.g. of pockets of cold gas with a wide spectrum of densities. Owing to their over-density, these blobs fall to the black hole.

If these these blobs have a significant amount of angular momentum, they cannot feed the black hole. On the other hand, low angular momentum blobs are allowed to accrete, igniting a fresh AGN outburst, which in turn disturbs the ICM, restarting the cycle with a new injection of blobs.

In this duty cycle some of the gas cools to low temperatures $\left(\lesssim 10^{4} \mathrm{~K}\right)$ before the next major heating episode, while the rest is heated back to a relatively high temperature. The presence of a detectable amount of gas cooling below X-ray emitting temperatures is a specific prediction of this model. Indeed, in the CF cluster A 2597 both extreme-UV and X-ray observations indicate a mass cooling rate of $\sim 100 M_{\odot} \mathrm{yr}^{-1}$, which is $\sim 0.2$ of the value quoted in the past based on ROSAT X-ray observations (see the discussion in Morris \& Fabian, 2005). In the CF cluster A 2029, Clarke et al. (2004) find a substantial amount of gas at a temperature of $\approx 10^{6} \mathrm{~K}$; a $\mathrm{CF}$ model gives a mass cooling rate of $\sim 50 M_{\odot} \mathrm{yr}^{-1}$.

According to the results of Pizzolato \& Soker (2005) and Soker (2006), a key role in the cold bubbles' accretion and feedback is played by the ICM entropy profile.

Long after an outburst, following an extended period of cooling, the entropy profile is steep. In this case it is difficult for an infalling blob to accrete and feed the black hole: on its way it will reach an equilibrium radius where its density equals that of its surroundings; it then dissolves before accreting on the black hole. Only the blobs the birthplace of which has a small entropy difference with respect to the core can accrete. Therefore, the first accretion episodes are most likely to involve small blobs, stemming not far from the centre. The AGN activity induced by their accretion is rather weak, and only raises the entropy very close to the AGN. In the meantime, the ICM further out keeps cooling, reducing the cooling time of the dense blobs. In addition, the combined action of cooling the far regions and heating the central one flattens the cluster's entropy profile. By the same token, due to the flat entropy profile now even far blobs may accrete on the AGN, triggering a major outburst. This may therefore heat the cluster on large scales.

There are four hurdles the blobs must overcome before accreting. 
1. Some pockets may be engulfed by the expanding radio lobes. As Pizzolato \& Soker (2005) (and references therein) argue, if the blobs are initially dense enough (say, 10 - 100 times the ambient medium) they can survive the shock, and accrete unhindered through the radio lobes.

2. These blobs must withstand the ICM thermal conduction: if it is too efficient, it would be able to evaporate these cold clouds before they can accrete on the AGN. We find from Figure 3 of Nipoti \& Binnev (2004) that the effective heat conduction should be $\lesssim 10^{-3}$ times the nominal Spitzer (1956) not to evaporate a blob of radius $a \sim 10-100 \mathrm{pc}$. Such a strong suppression factor is supported by theoretical considerations (Pistinner et al., 1996; Nath, 2003). The possible existence of magnetic turbulence (Schekochihin et al., 2006) may also strongly affect the transport coefficients, including thermal conductivity. A somewhat suppressed conduction is also consistent with some recent observations (M87: Molendi, 2002, NGC 5044: Buote et al., 2003).

3. As Nulsen (1986) pointed out, in the absence of a cohesive force a blob would be torn apart by the ram pressure in $\sim 10^{7} \mathrm{yr}$, i.e. a time considerably shorter than the time taken by the blob to fall to the centre. Some kind of cohesive force (like a magnetic tension) must then be at work to prevent the blob disruption.

4. If the blobs' angular momentum is too high, they are prevented from approaching the central black hole: the flux would merely stagnate, cool down and condense in filaments or stars, and the AGN fuelling is cut off altogether, thus making the feedback impossible. Indeed, the existence of a circumnuclear disc with radius $R_{d} \approx 10^{2}$ pc around M87 (Harms et al., 1994; Ford et al., 1994) shows that the flow possesses an amount of angular momentum.

However, the blobs are expected to form and accrete only in a region of the same extension as the inner gas entropy plateau $(\sim 5-30 \mathrm{kpc})$. The circularisation radius of this flow is expected to be of the same order as the actual size of the circumnuclear disc of M87 (Pizzolato \& Soker, $2005)$, i.e. direct accretion is possible down to the immediate vicinity of the black hole. In addition, the blobs may stem directly from ICM disturbances driven by an early AGN activity, but also from galaxies mass-stripping (Soker et al., 1991). Since the galaxies do not have an ordered bulk motion, also the blobs stripped from them are also unlikely to organise in an ordered flow with high net angular momentum.

So, to summarise, under some reasonable assumptions the cold blobs are able to survive long enough to accrete on the AGN and hence provide feedback.

The dense blobs that sink to the centre feed the AGN. The feedback is with the entire cool inner region, and not only with the gas close to the black hole. Any over-cooling taking place in the inner region, where the temperature profile is flat, will lead to many small and dense blobs, which feed the AGN. 
A Bondi accretion radius as large as the disc around the black hole of M87 further suggests that the simple Bondi accretion flow (Churazov et al., 2002; Nulsen, 2004) does not hold; the accreted material has a larger angular momentum, and may come from much larger radii. Also for cluster A 1835 the Bondi accretion is unlikely to be the main engine powering the feedback (McNamara et al., 2006).

\section{Summary}

We propose that the feedback occurs with the entire cool inner region, $r \lesssim$ $5-30 \mathrm{kpc}$, in what we term a cold-feedback model. In the proposed scenario non-linear over-dense blobs of gas cool fast and are removed from the ICM before the next major AGN heating event in their region. It is important to note that an AGN burst can take place and heat other regions, since the jets and/or bubbles may expand in other directions as well. The typical interval between such heating events at a specific region is $\approx 10^{8}$ yr. Some of these blobs cool and sink toward the central black hole, while others may form stars and cold molecular clouds.

Four conditions should be met in the inner region participating in the feedback heating.

1. In order for the blob not to reach an equilibrium point before accreting, a shallow ICM entropy profile is required. The relevant dense blobs then must form within the cluster core, typically $\lesssim 5-30 \mathrm{kpc}$ from the centre. We note that the lower segment of magnetic flux loops can be prevented from reaching the stabilising point by the upward force of the magnetic tension inside the loop (Soker, 2004). Therefore, some perturbations can be formed at large distances, where density profile is steep, and still cool to low temperature and feed the central black hole.

2. Non-linear perturbations are required. These presumably formed mainly by previous AGN activity, e.g. jets and radio lobes.

3. The cooling rate of these non-linear perturbations is short relative to few times the typical interval between successive AGN outbursts.

4. The blobs must not be evaporated by thermal conduction before they are delivered to the AGN. This requires a strong suppression of thermal conduction.

The first and the third condition, which are not completely independent of each other, require that the initial ICM cools by a factor of a few before the feedback starts operating, and the second condition requires that the inner region must be disturbed.

The cold-feedback model has the following implications and predictions

1. The optical filaments observed in many CF-clusters and the cooler molecular gas detected via $\mathrm{CO}$ observations come from cooling ICM (with some amount possibly from stripping from galaxies). 
2. Some X-ray emission from gas at temperatures $\lesssim 10^{7} \mathrm{~K}$ is expected, consistent with the moderate CF model. This is much more than in many other AGN heating models, but at least an order of magnitude below what predicted by the old CF model, We stress that in the cold-feedback heating, cooling flows do exist. Such gas cooling to below X-ray emitting temperatures was found recently in two CF clusters (A2597: Morris \& Fabian 2005; A2029: Clarke et al. 2004).

3 . The feeding of the central black hole with cold gas in the cold feedback models makes the process similar in some aspects to that of AGN in spiral galaxies. Therefore, the outflow can be similar (Soker \& Pizzolato, 2005).

4. It is possible that in the cold feedback model a substantial fraction of gas that cooled to low temperatures and was accreted to the accretion disc around the central black hole, is injected back to the ICM at nonrelativistic velocities (Soker \& Pizzolato, 2005).

\section{References}

Begelman, M. C. 2004, in Coevolution of Black Holes and Galaxies, ed. L. C. Ho, 374-+

Blanton, E. L., Sarazin, C. L., McNamara, B. R., \& Wise, M. W. 2001, ApJ, $558, \mathrm{~L} 15$

Buote, D. A., Lewis, A. D., Brighenti, F., \& Mathews, W. G. 2003, ApJ, 594, 741

Churazov, E., Sunyaev, R., Forman, W., \& Böhringer, H. 2002, MNRAS, 332, 729

Clarke, T. E., Blanton, E. L., \& Sarazin, C. L. 2004, ApJ, 616, 178

Fabian, A. C. 1994, ARA\&A, 32, 277

Ford, H. C., Harms, R. J., Tsvetanov, Z. I., Hartig, G. F., Dressel, L. L., Kriss, G. A., Bohlin, R. C., Davidsen, A. F., Margon, B., \& Kochhar, A. K. 1994, ApJ, 435, L27

Harms, R. J., Ford, H. C., Tsvetanov, Z. I., Hartig, G. F., Dressel, L. L., Kriss, G. A., Bohlin, R., Davidsen, A. F., Margon, B., \& Kochhar, A. K. 1994, ApJ, 435, L35

McNamara, B. R., Rafferty, D. A., Bîrzan, L., Steiner, J., Wise, M. W., Nulsen, P. E. J., Carilli, C. L., Ryan, R., \& Sharma, M. 2006, ApJ, 648, 164

Molendi, S. 2002, ApJ, 580, 815

Morris, R. G. \& Fabian, A. C. 2005, MNRAS, 358, 585

Nath, B. B. 2003, MNRAS, 340, L1

Nipoti, C. \& Binney, J. 2004, MNRAS, 349, 1509

Nulsen, P. 2004, in The Riddle of Cooling Flows in Galaxies and Clusters of galaxies, ed. T. Reiprich, J. Kempner, \& N. Soker, 259-262

Nulsen, P. E. J. 1986, MNRAS, 221, 377

Peterson, J. R. \& Fabian, A. C. 2006, Physics Reports, 427, 1 
Pistinner, S., Levinson, A., \& Eichler, D. 1996, ApJ, 467, 162

Pizzolato, F. \& Soker, N. 2005, ApJ, 632, 821

Schekochihin, A. A., Cowley, S. C., \& Dorland, W. 2006, astro-ph/0610810

Soker, N. 2004, MNRAS, 350, 1015

-. 2006, New Astronomy, 12, 38

Soker, N., Bregman, J. N., \& Sarazin, C. L. 1991, ApJ, 368, 341

Soker, N. \& Pizzolato, F. 2005, ApJ, 622, 847

Spitzer, L. 1956, Physics of Fully Ionized Gases (Interscience Publishers, New York) 